\title{
Exergames como ferramenta de promoção de atividade física em crianças: uma
}

\section{revisão integrativa}

\author{
Exergames as a tool to promote physical activity in children: an integrative review \\ Exergames como herramienta para promover la actividad física en los niños: una revisión \\ integradora
}

Recebido: 29/12/2021 | Revisado: 03/01/2022 | Aceito: 07/01/2022 | Publicado: 11/01/2022

Alison Brendo Cintra de Souza
ORCID: https://orcid.org/0000-0001-6233-6982
Centro Universitário da Grande Dourados, Brasil
E-mail: alisonbrendo7@ gmail.com
Joel Saraiva Ferreira
ORCID: https://orcid.org/0000-0003-4437-3100
Universidade Federal de Mato Grosso do Sul, Brasil
E-mail: falecomjoel@hotmail.com
Luis Eduardo Moraes Sinésio
ORCID: https://orcid.org/0000-0002-3839-1049
Instituto Federal de Educação, Ciência e Tecnologia de Mato Grosso do Sul, Brasil
Centro Universitário da Grande Dourados, Brasil
E-mail: luis.sinesio@ unigran.br
Fabiano Rodrigues Pissurno
ORCID: https://orcid.org/0000-0002-7290-8208
Centro Universitário da Grande Dourados, Brasil
E-mail: fabiano.pissurno@unigran.br
Gildiney Penaves de Alencar
ORCID: https://orcid.org/0000-0002-5177-495X
Universidade Federal de Mato Grosso do Sul, Brasil
Centro Universitário da Grande Dourados, Brasil
E-mail: gildiney.gpa@gmail.com

\begin{abstract}
Resumo
O objetivo desta pesquisa foi descrever os benefícios dos exergames como ferramenta de promoção de atividade física em crianças. Trata-se de uma revisão integrativa de artigos publicados nas bases de dados Lilacs, SciELO e PubMed, no período de 2011 a 2021, selecionados por meio dos descritores exatos e sinônimos em português e em inglês dos termos "exergames", "atividade física" e "crianças". Foram incluídos artigos com texto completo disponível em meio online; de forma gratuita; nos idiomas português, inglês ou espanhol; que respondessem à questão norteadora e; tratassem de estudos experimentais ou revisões de literatura que buscaram analisar os exergames como ferramenta de atividade física em crianças (até 11 anos). A amostra final foi composta por oito artigos. Todos os estudos constataram que o aumento do nível de atividade física foi o principal benefício da prática de exergames, além de aumentar a motivação, o gasto energético, a frequência cardíaca, melhorar a composição corporal, medidas antropométricas e a saúde de forma geral, principalmente nos jogos que envolvem a prática de esportes (Wii Sports), a dança (Dance Dance Revolution e Just Dance) e os exercícios físicos (Wii Fit). Os exergames se mostram como ferramenta efetiva na promoção de atividade física em crianças. Entretanto, é importante que sejam utilizados de forma a complementar as atividades físicas convencionais, para que se tenha um melhor aproveitamento de todos os seus benefícios e estimulem hábitos de vida mais saudáveis.
\end{abstract}

Palavras-chave: Exergames; Atividade física; Crianças.

\begin{abstract}
The aim of this research was to describe the benefits of exergames as a tool to promote physical activity in children. This is an integrative review of articles published in Lilacs, SciELO and PubMed databases, from 2011 to 2021, selected using exact descriptors and synonyms in Portuguese and English for the terms "exergames", "physical activity" and "children". Articles with full text available online were included; for free; in Portuguese, English or Spanish; to answer the guiding question and; dealt with experimental studies or literature reviews that sought to analyze exergames as a physical activity tool in children (up to 11 years). The final sample consisted of eight articles. All studies found that the increase in the level of physical activity was the main benefit of the practice of exergames, in addition to increasing motivation, energy expenditure, heart rate, improving body composition, anthropometric measures and health in general, especially in games that involve the practice of sports (Wii Sports), dancing (Dance
\end{abstract}


Dance Revolution and Just Dance) and physical exercises (Wii Fit). Exergames are an effective tool in promoting physical activity in children. However, it is important that they be used in a way that complements conventional physical activities, so that you can have a better use of all their benefits and encourage healthier lifestyle habits.

Keywords: Exergames; Physical activity; Children.

\section{Resumen}

El objetivo de esta investigación fue describir los beneficios de los exergames como herramienta para promover la actividad física en los niños. Se trata de una revisión integradora de artículos publicados en las bases de datos Lilacs, SciELO y PubMed, de 2011 a 2021, seleccionados utilizando descriptores exactos y sinónimos en portugués e inglés para los términos "exergames", "actividad física" y "niños". Se incluyeron artículos con texto completo disponible en línea; gratis; en portugués, inglés o español; para responder a la pregunta de orientación y; abordó estudios experimentales o revisiones de la literatura que buscaban analizar los exergames como herramienta de actividad física en los niños (hasta los 11 años). La muestra final estuvo formada por ocho artículos. Todos los estudios encontraron que el aumento en el nivel de actividad física era el principal beneficio de la práctica de exergames, además de incrementar la motivación, el gasto energético, la frecuencia cardíaca, mejorar la composición corporal, las medidas antropométricas y la salud en general, especialmente en los juegos que involucran la práctica de deportes (Wii Sports), baile (Dance Dance Revolution y Just Dance) y ejercicios físicos (Wii Fit). Los Exergames son una herramienta eficaz para promover la actividad física en los niños. Sin embargo, es importante que se utilicen de forma que complementen las actividades físicas convencionales, para que puedas aprovechar mejor todos sus beneficios y fomentar hábitos de vida más saludables.

Palabras clave: Exergames; Actividad física; Niños.

\section{Introdução}

A utilização das mídias digitais pelas crianças (6 a 11 anos) se apresenta numa constante evolução e atrai este público devido à ludicidade e interação que essas ferramentas proporcionam, principalmente quando envolvem uma relação de ensino e aprendizagem (Abreu et al., 2018; Silva, Fagundes, \& Menezes, 2018). Uma dessas ferramentas que vem chamando a atenção do público infantil são os exergames, que, mesmo sendo mais utilizados nas escolas (Bittencourt et al., 2018), também são populares fora deste ambiente e proporcionam diversos efeitos favoráveis, como a melhora das habilidades e das capacidades motoras dos praticantes (Medeiros et al., 2017), fatores que influenciam positivamente no desenvolvimento motor (Silva et al., 2018) e na própria corporeidade (Alencar et al., 2019).

Os exergames são jogos ativos que permitem a movimentação corporal durante a prática do jogo de videogame e exigem do praticante certo nível de gasto energético. Por isso, são considerados uma forma de aliar a atividade física com a tecnologia (Lin, 2015). Estes jogos possuem um sistema de captação dos movimentos realizados pelo praticante e os tornam parte do jogo, conciliando o desempenho na atividade virtual com as ações motoras (Deutsch et al., 2011; Peres et al., 2021).

Para que as crianças possam desfrutar dos benefícios da prática de atividade física para a saúde, a Organização Mundial da Saúde (OMS) recomenda que façam ao menos 60 minutos de atividade moderada a vigorosa durante todos os dias da semana (principalmente aeróbica), assim como aquelas que fortaleçam os músculos e os ossos por pelo menos três dias, além de reduzir o comportamento sedentário diário (World Health Organization, 2020). Essas orientações são ratificadas pelo Guia de Atividade Física para a População Brasileira (Brasil, 2021).

Como uma forma de cumprir tais recomendações, os exergames se mostram como uma excelente alternativa por prender a atenção das crianças e despertar o interesse na realização das atividades físicas (Lins et al., 2020), inclusive em períodos com restrições relacionadas à interação social e mobilidade urbana, tal como vivenciado pela população mundial durante a pandemia de COVID-19 (Florêncio Júnior et al., 2020).

Ademais, os exergames podem atuar positivamente no combate à obesidade infantil, devido ao aumento do gasto energético diário produzido com a prática destes jogos (Graf et al.,2009; Dias et al., 2017), além de promover a melhora da autoestima e da imagem corporal (Aragão, 2018; Aragão et al., 2020), pois esses fatores são constantemente influenciados pelas experiências pessoais e pela imagem que a criança tem de si (Papalia et al., 2006). No entanto, o conjunto de benefícios obtidos pelas crianças que praticam regularmente exergames ainda não está completamente descrito na literatura científica. 
Neste sentido, este estudo teve como objetivo descrever os benefícios dos exergames como ferramenta de promoção de atividade física em crianças.

\section{Metodologia}

O presente estudo se caracteriza como uma revisão integrativa da literatura e seguiu rigorosamente as recomendações de Whittemore e Knafl (2005) em relação aos cinco passos para a sua realização (Figura 1).

Figura 1 - Passos da revisão integrativa segundo Whittemore e Knafl (2005).

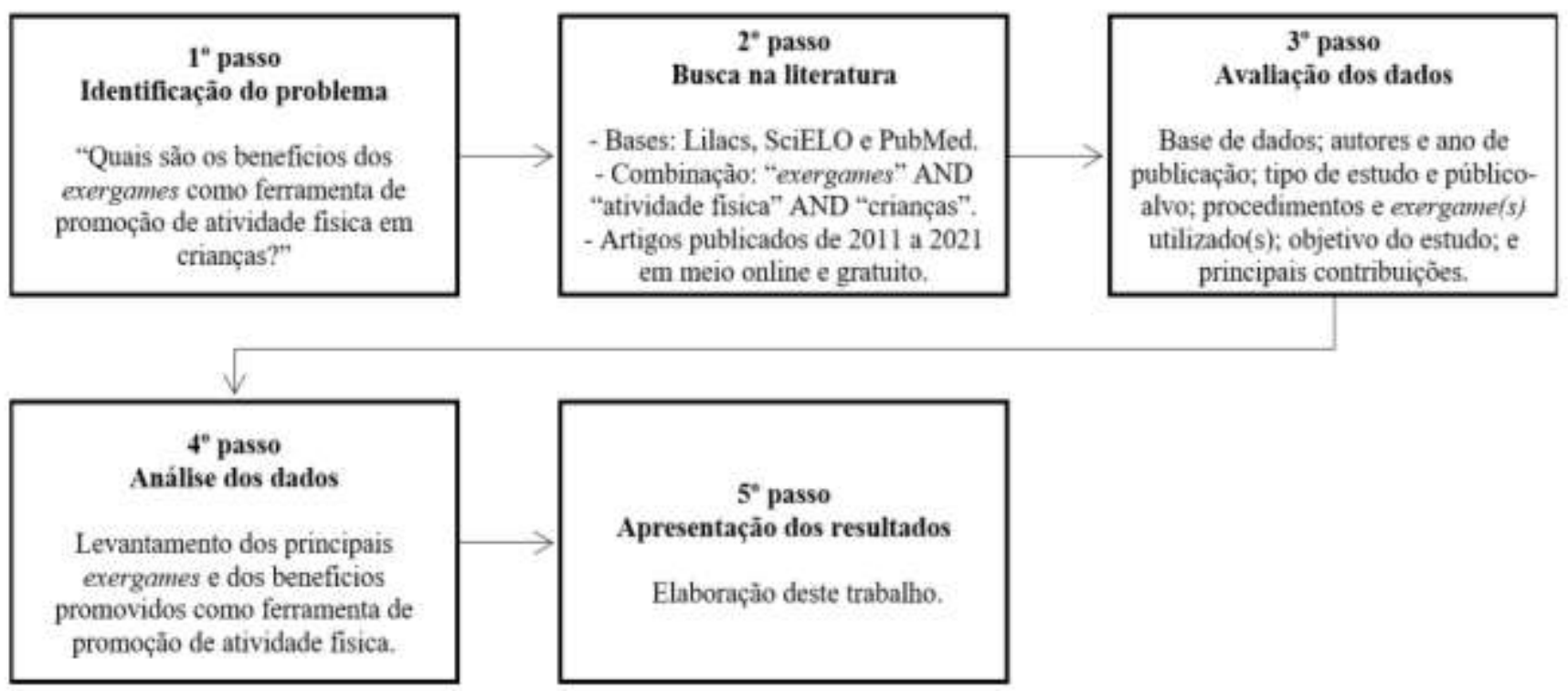

Fonte: Autores (2022).

O problema de pesquisa (primeiro passo) foi definido por meio da questão norteadora: "Quais são os benefícios dos exergames como ferramenta de promoção de atividade física em crianças?". Posteriormente, a busca na literatura (segundo passo), conduzida por dois revisores independentes e às cegas, ocorreu nas bases de dados Lilacs, SciELO e PubMed, por meio das combinações dos descritores exatos e sinônimos, com utilização do operador booleano AND da seguinte forma: "exergames" AND "atividade física" AND "crianças" (em português); "exergames" AND "physical activity" AND "children" (em inglês).

Como critérios de inclusão, foram estabelecidos os seguintes filtros de busca: artigos com texto completo disponível em meio online; de forma gratuita; nos idiomas português, inglês ou espanhol; publicados de 2011 a 2021; que respondessem à questão norteadora e; tratassem de estudos experimentais ou revisões de literatura que buscaram analisar os exergames como ferramenta de promoção de atividade física em crianças, sendo excluídos os artigos publicados fora do período préestabelecido, estudos duplicados e aqueles conduzidos com o público-alvo distinto às crianças. O período de estudo (2011 a 2021) foi selecionado em função da maior evolução dos estudos relacionados aos exergames como prática de atividade física e lançamento do Kinect para Xbox 360 (Ferreira, \& Francisco, 2017).

Ainda como parte do segundo passo, foi conduzida a pré-seleção dos artigos, com a leitura do título, resumo e palavras-chave. Os artigos pré-selecionados foram lidos na íntegra e apenas os que cumpriram com todos os critérios de inclusão foram utilizados no passo seguinte. 
Para avaliar os dados obtidos dos artigos selecionados (terceiro passo), foi elaborado um instrumento de coleta de dados contendo as seguintes variáveis de análise: base de dados; autores e ano de publicação; tipo de estudo e público alvo; procedimentos e exergame(s) utilizado(s); objetivo do estudo; e principais contribuições.

No quarto passo (análise dos dados), foi realizada uma categorização da amostra com uma abordagem da quantidade de artigos encontrados e a descrição de onde foram oriundos; uma avaliação dos artigos encontrados em busca de responder ao questionamento da pesquisa; além da discussão dos dados em torno do objetivo desta revisão, levantamento dos principais exergames e quais os benefícios identificados como ferramenta de promoção de atividade física.

Por fim, a última etapa (apresentação dos resultados) sucedeu na elaboração deste, que apresenta de forma sintetizada os resultados da revisão integrativa acerca dos benefícios dos exergames como ferramenta de promoção de atividade física em crianças.

\section{Resultados}

\subsection{Caracterização da amostra}

As buscas nas bases de dados resultaram em 217 artigos, sendo 50 publicações encontradas na base Lilacs, um artigo na SciELO e 166 na PubMed. Após a aplicação dos filtros, pré-seleção com a leitura dos títulos, resumos e palavras-chave, critérios de inclusão e exclusão, foram selecionados 20 artigos que foram lidos na íntegra, sendo excluídos 12 deles (sete por não possuírem o texto completo de forma gratuita, dois por não terem as crianças como público-alvo, dois por não responderem a questão norteadora e um por duplicação), resultando em uma amostra final de oito artigos, dos quais dois oriundos da base Lilacs e seis da PubMed (Figura 2).

Figura 2 - Fluxograma das etapas de seleção dos artigos.

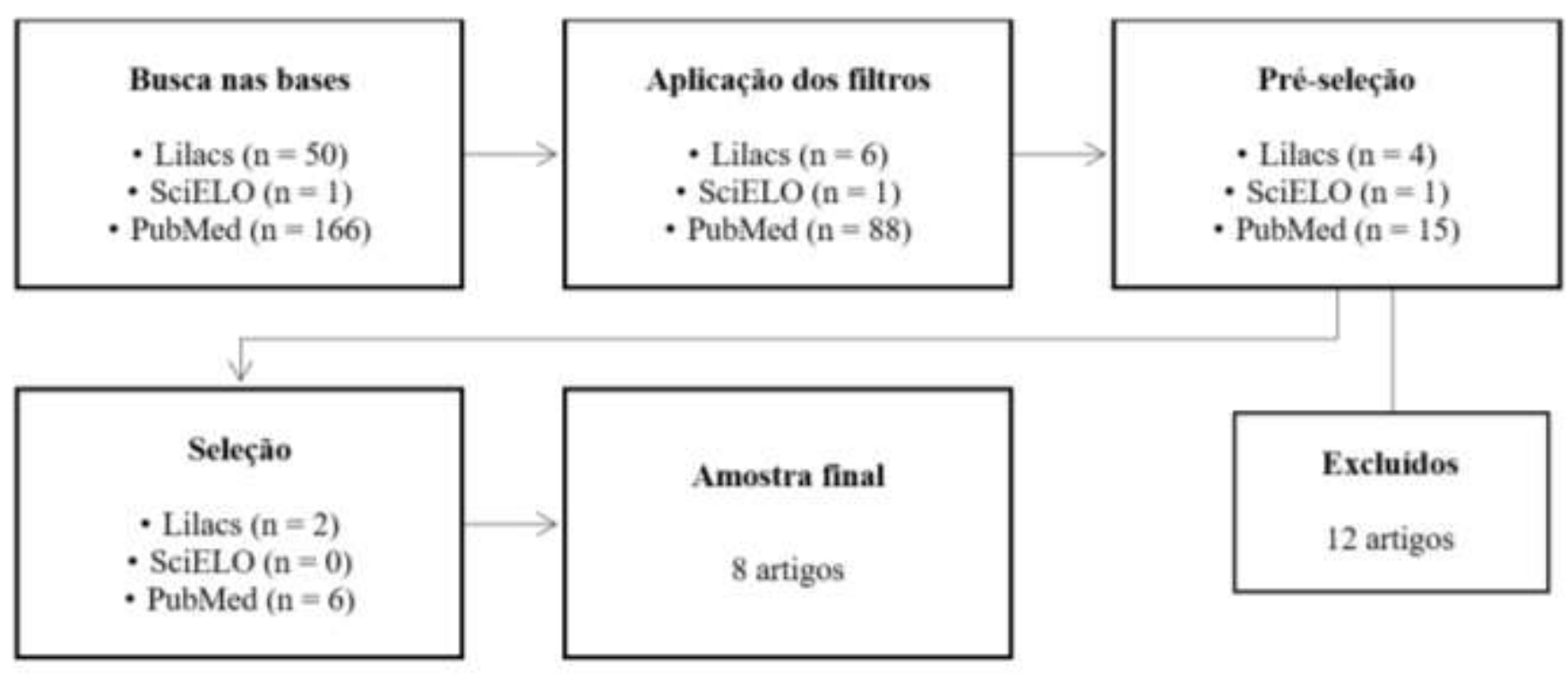

Fonte: Autores (2022).

\subsection{Avaliação dos artigos}

Para facilitar a compreensão da pesquisa, foram selecionados os artigos dentro de uma linha temporal de 10 anos (2011 a 2021), fato que possibilitou uma visão atualizada sobre os exergames como ferramenta de promoção de atividade física, pois a maioria dos artigos encontrados foram publicados entre 2017 e 2021 (62,5\%). No que tange ao tipo de estudo, cinco se trataram de revisões sistemáticas $(62,5 \%)$, dois experimentais $(25,0 \%)$ e uma revisão de literatura $(12,5 \%)$ com a 
aplicação da técnica SWOT $($ Strenghts = Forças; Weaknesses $=$ Fraquezas; Opportunities = Oportunidades; Threats $=$ Ameaças), o que permitiu uma análise diversificada da utilização dos exergames no contexto atual.

Grande parte dos artigos retrataram a escola como ambiente de uso dos exergames (37,5\%), mas outros contextos, como o âmbito hospitalar (12,5\%) também foram identificados. Os principais consoles utilizados foram o Nintendo Wii, Xbox e Sony Playstation. Já os exergames mais frequentes eram relacionados aos esportes $(75,0 \%)$, como o Wii Sports (principalmente os jogos de beisebol, tênis e boxe); à dança (62,5\%), como o Dance Dance Revolution (DDR) e o Just Dance; aos exercícios físicos (50,0\%), como o Wii Fit; e o Monster 4x4 (maior aumento do nível de atividade física), usados para combater o sedentarismo, sobrepeso e obesidade, de forma a complementar a atividade física convencional e incentivar a prática de exercício físico.

Os oito artigos que fizeram parte desta revisão integrativa foram avaliados de acordo com as seguintes variáveis: base de dados; autores e ano de publicação; tipo de estudo e público alvo; procedimentos e exergame(s) utilizado(s); objetivo do estudo; e principais contribuições (Quadro 1).

Quadro 1 - Avaliação dos artigos de acordo com a ordem cronológica de publicação.

\begin{tabular}{|c|c|c|c|c|c|}
\hline ڤ్ & $\begin{array}{l}\text { Autores e ano } \\
\text { de publicação }\end{array}$ & $\begin{array}{l}\text { Tipo de estudo e } \\
\text { público-alvo }\end{array}$ & $\begin{array}{c}\text { Procedimentos e } \\
\text { exergame }(s) \text { utilizado(s) }\end{array}$ & Objetivo do estudo & Principais contribuições \\
\hline $\begin{array}{l}\sum_{0}^{D} \\
\vec{D}\end{array}$ & $\begin{array}{l}\text { Shayne, Fogel, } \\
\text { Miltenberger e } \\
\text { Koehler (2012) }\end{array}$ & $\begin{array}{l}\text { - Experimental. } \\
\text { - } 4 \text { meninos da } \\
\text { terceira série de } \\
\text { uma escola } \\
\text { primária pública } \\
\text { da Florida, EUA. }\end{array}$ & $\begin{array}{l}\text { - Aulas de Educação Física } \\
\text { tradicional e aulas com } \\
\text { exergaming. } \\
\text { - Dance Dance Revolution } \\
\text { (DDR), Fit Interactive } 3 \text { Kick, } \\
\text { Madden Football, Monster } \\
\text { 4x4, Virtual Bike e Wii Sports } \\
\text { (Beisebol, Tênis ou Boxe). }\end{array}$ & $\begin{array}{l}\text { - Comparar os efeitos do } \\
\text { exergaming e da } \\
\text { educação física } \\
\text { tradicional na atividade } \\
\text { física e identificar as } \\
\text { máquinas associadas aos } \\
\text { níveis mais baixos e mais } \\
\text { altos de atividade física. }\end{array}$ & $\begin{array}{c}\text { - Aulas com exergaming produziram } \\
\text { porcentagens mais altas de atividade } \\
\text { física, porém, é preciso avaliar a sua } \\
\text { intensidade e os efeitos nos fatores } \\
\text { de saúde. } \\
\text { - O Monster } 4 \times 4 \text { e o DDR resultaram } \\
\text { nas maiores porcentagens de } \\
\text { atividade física, enquanto o Wii nas } \\
\text { menores. } \\
\text { - É necessário avaliar o custo e } \\
\text { benefício das máquinas. }\end{array}$ \\
\hline$\frac{\tilde{J}}{ב}$ & $\begin{array}{c}\text { Pereira, } \\
\text { Rodrigues, } \\
\text { Campos e } \\
\text { Amorim (2012) }\end{array}$ & $\begin{array}{c}\text { - Revisão } \\
\text { sistemática. } \\
\text { - Jovens com idade } \\
\text { igual ou inferior a } \\
18 \text { anos. }\end{array}$ & $\begin{array}{l}\text { - Busca na literatura, via } \\
\text { PubMed e Science Direct, de } \\
\text { artigos publicados entre } 2006 \\
\text { e } 2011 . \\
\text { - Wii Sports e Wii Fit, Sony } \\
\text { EyeToy, XaviX (boliche e J- } \\
\text { Mat) e Dance Dance } \\
\text { Revolution (DDR). }\end{array}$ & $\begin{array}{l}\text { - Analisar o gasto } \\
\text { energético (GE), a } \\
\text { frequência cardíaca (FC) } \\
\text { e o nível de atividade } \\
\text { física durante a prática de } \\
\text { exergames, comparando- } \\
\text { os ao repouso, as } \\
\text { atividades sedentárias e } \\
\text { às atividades físicas. }\end{array}$ & $\begin{array}{l}\text { - Aumento do GE, FC e nível de } \\
\text { atividade física e melhora na } \\
\text { composição corporal em comparação } \\
\text { às atividades sedentárias. } \\
\text { - Os exergames podem ser uma boa } \\
\text { opção para o aumento do GE e nível } \\
\text { de atividade física de crianças e } \\
\text { adolescentes, desde que sejam } \\
\text { associados a outros tipos de atividade } \\
\text { física. }\end{array}$ \\
\hline$\sum_{0}^{D}$ & $\begin{array}{l}\text { Lamboglia et } \\
\text { al. (2013) }\end{array}$ & $\begin{array}{c}\text { - Revisão } \\
\text { sistemática. } \\
\text { - Crianças e } \\
\text { adolescentes de } 6 \text { a } \\
15 \text { anos. }\end{array}$ & $\begin{array}{c}\text { - Busca na literatura, via } \\
\text { SciELO, LILACS, PubMed, } \\
\text { Ebsco e Science Direct, de } \\
\text { artigos publicados entre } 2008 \\
\text { e } 2012 . \\
\text { - Wii Sports, XaviX Bowling } \\
\text { e J-Mat, Jackie's Action Run } \\
\text { e Dance Dance Revolution } \\
\text { (DDR). }\end{array}$ & $\begin{array}{l}\text { - Avaliar a utilização do } \\
\text { exergaming como } \\
\text { ferramenta estratégica } \\
\text { para a promoção de } \\
\text { comportamentos } \\
\text { saudáveis no combate à } \\
\text { obesidade infantil. }\end{array}$ & $\begin{array}{l}\text { - O exergaming aumentou os níveis } \\
\text { de atividade física, gasto de } \\
\text { energia, consumo máximo de } \\
\text { oxigênio, frequência cardíaca e } \\
\text { reduziu a circunferência da cintura e } \\
\text { o tempo sedentário na tela. } \\
\text { - O exergaming pode ser uma } \\
\text { ferramenta para um estilo de vida } \\
\text { ativo e saudável, útil no combate à } \\
\text { obesidade infantil. }\end{array}$ \\
\hline
\end{tabular}




\begin{tabular}{|c|c|c|c|c|c|}
\hline$\frac{\tilde{g}}{\exists}$ & $\begin{array}{c}\text { Vaghetti, } \\
\text { Ferreira, } \\
\text { Cavalli, } \\
\text { Monteiro- } \\
\text { Junior e Del } \\
\text { Vecchio (2017) }\end{array}$ & $\begin{array}{l}\text { - Revisão } \\
\text { sistemática. } \\
\text { - Alunos em } \\
\text { ambiente escolar } \\
\text { ou selecionados } \\
\text { neste ambiente. }\end{array}$ & $\begin{array}{l}\text { - Busca na literatura, via } \\
\text { Science Direct, PubMed, } \\
\text { SciELO, EBSCO host, Health } \\
\text { Games Research, IEEE e Eric, } \\
\text { de estudos publicados entre } \\
2011 \text { e } 2017 . \\
\text { - Nintendo Wii (tênis, boliche, } \\
\text { boxe), X Box Kinect } \\
\text { (beisebol, futebol e tênis de } \\
\text { mesa). }\end{array}$ & $\begin{array}{l}\text { - Investigar e sintetizar os } \\
\text { achados de estudos que } \\
\text { desenvolveram ações no } \\
\text { ambiente escolar com a } \\
\text { utilização de exergames. }\end{array}$ & $\begin{array}{l}\text { - Os exergames podem aumentar os } \\
\text { níveis de atividade física, modificar } \\
\text { variáveis antropométricas, aumentar } \\
\text { a motivação e desempenho escolar. } \\
\text { - Professores de Educação Física } \\
\text { podem realizar a integração de } \\
\text { exergames com aulas tradicionais. }\end{array}$ \\
\hline $\begin{array}{l}\sum_{0}^{D} \\
\sum_{0}^{\infty}\end{array}$ & $\begin{array}{c}\text { Benzing e } \\
\text { Schmidt (2018) }\end{array}$ & $\begin{array}{l}\text { - Revisão de } \\
\text { literatura. } \\
\text { - Crianças e } \\
\text { adolescentes. }\end{array}$ & $\begin{array}{c}\text { - Aplicação da técnica SWOT } \\
\text { (Strenghts = Forças; } \\
\text { Weaknesses = Fraquezas; } \\
\text { Opportunities = } \\
\text { Oportunidades; Threats = } \\
\text { Ameaças). } \\
\text { - Exergames de forma geral. }\end{array}$ & $\begin{array}{l}\text { - Fornecer uma visão } \\
\text { sobre os pontos fortes } \\
\text { (PFor), fracos (PFr), } \\
\text { oportunidades (OP) e } \\
\text { ameaças (AM) dos } \\
\text { exergames para promover } \\
\text { atividade física em } \\
\text { crianças e adolescentes. }\end{array}$ & $\begin{array}{c}\text { - PFor: Aumento da motivação e o } \\
\text { envolvimento na atividade física. } \\
\text { - PFr: Potencial para aumentar a } \\
\text { atividade física é subexplorado. } \\
\text { - OP: Desenvolver os pontos fortes } \\
\text { para tornar as crianças e adolescentes } \\
\text { mais ativos. } \\
\text { - AM: Substituição dos exercícios } \\
\text { físicos tradicionais e aumento do } \\
\text { tempo de tela. }\end{array}$ \\
\hline 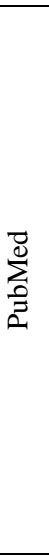 & $\begin{array}{l}\text { Ramírez- } \\
\text { Granizo, } \\
\text { Ubago- } \\
\text { Jiménez, } \\
\text { González- } \\
\text { Valero, } \\
\text { Puertas-Molero } \\
\text { e Román-Mata } \\
\text { (2020) }\end{array}$ & $\begin{array}{l}\text { - Revisão } \\
\text { sistemática. } \\
\text { - Crianças em } \\
\text { idade escolar. }\end{array}$ & $\begin{array}{c}\text { - Busca na literatura, via Web } \\
\text { of Science, de estudos } \\
\text { publicados entre 2016 e } 2020 \\
\text { com emprego do PRISMA. } \\
\text { - Wii Sports e Wii Fit, Just } \\
\text { Dance. }\end{array}$ & $\begin{array}{l}\text { - Realizar uma revisão } \\
\text { sistemática da literatura } \\
\text { científica abordando o } \\
\text { efeito da prática de } \\
\text { atividade física e o uso de } \\
\text { exergames por meio de } \\
\text { estudos longitudinais e } \\
\text { experimentais. }\end{array}$ & $\begin{array}{l}\text { - Desempenho cognitivo e } \\
\text { velocidade de reação são } \\
\text { beneficiados pela prática de atividade } \\
\text { física com exergames. } \\
\text { - Os exergames promovem uma } \\
\text { melhoria em termos de motivação, } \\
\text { estado de saúde e participação. } \\
\text { - O uso de videogames ativos é um } \\
\text { elemento inovador para incentivar a } \\
\text { prática de atividade física desde } \\
\text { cedo, desde que complementada por } \\
\text { atividades frequentes ou intensas. }\end{array}$ \\
\hline $\begin{array}{l}\sum_{0}^{D} \\
\equiv \\
\equiv\end{array}$ & $\begin{array}{l}\text { González- } \\
\text { González, Del } \\
\text { Rio, Toledo- } \\
\text { Delgado e } \\
\text { García-Peñalvo } \\
\quad(2021)\end{array}$ & $\begin{array}{l}\text { - Experimental. } \\
\text { - Crianças entre } 6 \\
\text { e } 12 \text { anos com } \\
\text { diagnóstico de } \\
\text { obesidade / } \\
\text { diabetes tipo II } \\
\text { atendidas em um } \\
\text { hospital. } \\
\end{array}$ & $\begin{array}{l}\text { - Programa PROVITAO: } \\
\text { sessões com videogame ativo } \\
\text { comercial, jogos ativos } \\
\text { esportivos e videogame ativo } \\
\text { com sensores biométricos. } \\
\text { - Wii Fit Plus, Jogos motores } \\
\text { no campo esportivo, TANGO: } \\
\text { H. }\end{array}$ & $\begin{array}{l}\text { - Descrever duas soluções } \\
\text { baseadas em jogos ativos } \\
\text { utilizando o sensor Kinect } \\
\text { e sensores biométricos, } \\
\text { projetadas para o } \\
\text { tratamento ambulatorial } \\
\text { da obesidade infantil. }\end{array}$ & $\begin{array}{l}\text { - As crianças desejavam frequentar } \\
\text { sessões semanais, motivados por } \\
\text { brincar, estar com colegas, aprender } \\
\text { e ganhar pontos. } \\
\text { - O programa foi eficaz na percepção } \\
\text { de aprendizagem de hábitos } \\
\text { saudáveis, hábito de atividade física } \\
\text { e dieta. }\end{array}$ \\
\hline $\begin{array}{l}\sum_{0}^{D} \\
\sum_{0}^{\infty}\end{array}$ & $\begin{array}{l}\text { Valeriani et al. } \\
\qquad(2021)\end{array}$ & $\begin{array}{c}\text { - Revisão } \\
\text { sistemática. } \\
\text { - Crianças e } \\
\text { adolescentes com } \\
\text { sobrepeso e } \\
\text { obesidade na faixa } \\
\text { etária de } 0 \text { a } 18 \\
\text { anos. }\end{array}$ & $\begin{array}{l}\text { - Busca na literatura, via } \\
\text { PubMed, Web of Science e } \\
\text { SPORTDiscus, de estudos } \\
\text { publicados sem limitação de } \\
\text { tempo até } 28 \text { de fevereiro de } \\
2021 \text { com emprego da } \\
\text { estrutura PICOS. } \\
\text { - Sony PlayStation Eye-Toy, } \\
\text { Xbox } 360 \text { Kinect, Dance } \\
\text { Dance Revolution (DDR), } \\
\text { Kinect Sports. }\end{array}$ & $\begin{array}{l}\text { - Avaliar o possível papel } \\
\text { dos exergames na } \\
\text { redução dos resultados } \\
\text { relacionados ao peso } \\
\text { entre crianças e/ou } \\
\text { adolescentes com } \\
\text { sobrepeso e/ou obesidade. }\end{array}$ & $\begin{array}{l}\text { - Possível efeito positivo dos } \\
\text { videogames ativos no peso em } \\
\text { crianças e adolescentes obesos. } \\
\text { - Os exergames podem representar } \\
\text { uma ferramenta útil no combate ao } \\
\text { sedentarismo e na promoção de } \\
\text { atividade física. }\end{array}$ \\
\hline
\end{tabular}

Fonte: Autores (2022). 


\section{Discussão}

No mapeamento dos trabalhos acerca da temática dos exergames, os pesquisadores foram unânimes em relação ao aumento da atividade física como principal benefício obtido na prática desses jogos, além de aumentar a motivação, o gasto energético, a frequência cardíaca, melhorar a composição corporal, medidas antropométricas e a saúde de forma geral, principalmente no Monster 4x4, nos jogos que envolvem a prática de esportes (Wii Sports), a dança (Dance Dance Revolution e Just Dance) e os exercícios físicos (Wii Fit).

Todos os benefícios supramencionados se tornam ainda mais relevantes quando a Organização Mundial da Saúde indica que mais de $80 \%$ da população adolescente mundial é insuficientemente ativa fisicamente e um em cada quatro adultos não atinge os níveis de atividade física recomendados (World Health Organization, 2020). Sendo assim, vislumbra-se uma possibilidade de modificação deste cenário, por meio do estímulo da prática de atividade física desde a infância, tendo os exergames como uma ferramenta eficaz para atingir esse objetivo (Pereira et al., 2012; Shayne et al.,; Lamboglia et al., 2013; Vaghetti et al., 2017; Benzing, \& Schmidt, 2018; Ramírez-Granizo et al., 2020; González-González et al., 2021; Valeriani et al., 2021).

$\mathrm{O}$ fato dos exergames mais utilizados serem aqueles relacionados aos esportes (como o beisebol, tênis ou boxe - Wii Sports) ou à dança (como o Dance Dance Revolution - DDR ou Just Dance), mostra uma tendência que se mantém desde o início do século XXI (Peng et al., 2012), com maior preferência do público infantil pelos jogos baseados nos movimentos de dança e prática de esportes. Neste sentido, os profissionais envolvidos com a atividade física devem estar atentos à demanda $\mathrm{e}$ às preferências das crianças, para que possam aproveitar de todos os seus benefícios, principalmente no que diz respeito ao potencial para aumentar a atividade física habitual desse público, fato considerado um ponto fraco por Benzing e Schmidt (2018).

Ainda, é necessário considerar quais jogos proporcionam um maior envolvimento com a atividade física para que o seu uso seja programado de acordo com a necessidade de cada criança, como indicam Shayne, Fogel, Miltenberger e Koehler (2012). Dentre os jogos mencionados pelas publicações analisadas no presente estudo, o Monster 4x4 e o DDR resultaram nas maiores porcentagens de atividade física, enquanto o Wii nas menores. Sendo assim, a escolha dos jogos também pode ser planejada para cada contexto e cada objetivo, em função do nível de atividade física desejada por quem desenvolve intervenções com exergames.

Quando comparados com os videogames tradicionais ou com atividades consideradas sedentárias, o uso dos exergames representa uma ferramenta importante, pois eleva o gasto energético, frequência cardíaca, consumo máximo de oxigênio, reduz a circunferência da cintura, o tempo sedentário na tela e interfere diretamente na composição corporal (Pereira et al.,; Lamboglia et al., 2013), condições favoráveis para a melhoria do estado de saúde (Ramírez-Granizo et al., 2020) e no combate à obesidade infantil (Lamboglia et al., 2013; Valeriani et al., 2021).

A melhora do desempenho cognitivo, aumento da motivação e do desempenho escolar também são benefícios apontados por Vaghetti et al. (2017) e Ramírez-Granizo et al. (2020), elementos positivos que sustentam a utilização dos exergames. Aragão et al. (2020) aponta ainda as vantagens obtidas na autoestima e na imagem corporal das crianças. Outro ponto importante trata-se de auxiliar aquelas crianças que apresentam dificuldade ou constrangimento em praticar atividade física em lugar público, pois estes jogos podem ser realizados no conforto da própria residência, sem necessidade da exposição (Medeiros et al., 2017; Kowaluk, \& Woźniewski, 2019) e também uma prática segura levando em conta a prevenção da transmissão do coronavírus causador da COVID-19 (Florêncio Júnior et al., 2020). Ou seja, as intervenções com exergames são amplas e ultrapassam os benefícios relacionados aos componentes biológicos do corpo humano.

Apesar das evidências apontarem uma multiplicidade de benefícios em relação ao uso dos exergames, Benzing e Schmidt (2018) destacam que a grande ameaça desses jogos é a substituição dos exercícios físicos tradicionais e o aumento do 
tempo de tela, fatores que devem ser constantemente observados para que os exergames não se tornem instrumentos que tirem as crianças do mundo real. Para isso, Pereira et al. (2012) e Ramírez-Granizo et al.(2020) sugerem que esses jogos devem ser utilizados como uma forma de chamar a atenção e estimular as crianças à prática de atividades físicas e precisam ser complementados por atividades físicas ou esportivas de maior intensidade (Marin et al., 2019).

Enfim, o papel principal dos exergames é motivar as crianças, incentivando-as a adotarem uma vida mais ativa e saudável, principalmente por meio da substituição dos comportamentos sedentários, como o excessivo tempo sentado. É um elemento benéfico e inovador que desenvolve a aprendizagem cognitiva, auxilia nos elementos socioculturais, psicossociais e promove hábitos de vida mais saudáveis (Barbosa et al., 2021; González-González et al., 2021). Mas, ainda assim, requer a complementaridade da prática de atividades físicas convencionais em outros momentos.

\section{Considerações Finais}

Os exergames promovem diversos benefícios, quando utilizados como ferramenta de promoção de atividade física em crianças, tais como o aumento do gasto energético, redução do peso corporal, desenvolvimento de habilidade cognitiva, propensão à adoção de hábitos saudáveis e incentivo à prática de atividade física convencional.

Dentre os principais exergames utilizados se destacam o Dance Dance Revolution (DDR) e o Just Dance, o Monster 4x4 e o Wii Sports (beisebol, tênis ou boxe), os quais são utilizados de forma virtual por meio dos consoles que possuem sensores de movimentos, óculos de realidade virtual e tapetes de dança que oferecem ao indivíduo uma proposta, metas ou objetivos a se cumprir.

Os exergames se mostram como ferramenta efetiva na promoção de atividade física em crianças. Entretanto, é importante que sejam utilizados de forma a complementar as atividades físicas convencionais, para que os benefícios voltados à saúde sejam aproveitados ao longo da vida.

Apesar das evidências já produzidas, novas pesquisas ainda são necessárias, principalmente para identificar a potencialidade dos exergames na promoção de atividade física em crianças em outros ambientes, além do contexto escolar. Outra demanda para investigação futura é o detalhamento dos jogos e dos consoles que mais se associam com a adesão das crianças às atividades físicas convencionais.

\section{Referências}

Abreu, C. A., Rosa, J. C. S., \& Matos, E. S. (2018). Usabilidade de aplicativos móveis educacionais infantis: design e avaliação de interação do Fantastic Pirates. Revista Novas Tecnologias na Educação, 16 (1), 1-10. https://doi.org/10.22456/1679-1916.86042

Alencar, G. P., Pereira, M. G. L., Pereira, T. T., Oliveira, C. M. V., Morais, C. S., \& Ota, G. E. (2019). La corporeidad y sus relaciones con la Educación Física escolar. Lecturas: Educación Física y Deportes, 24 (252), 154-164. https://www.efdeportes.com/efdeportes/index.php/EFDeportes/article/view/856

Aragão, R. B. (2018). Efeitos de exergames na autoestima, imagem corporal e antropometria de crianças com sobrepeso/obesidade [Dissertação de Mestrado em Educação Física e Esporte]. Universidade de São Paulo, Ribeirão Preto. https://teses.usp.br/teses/disponiveis/109/109131/tde-17082018$164231 /$ publico/segunda.pdf

Aragão, R. B., Dias, J. D., Alípio, T., Pinto, R. P., Libertore Junior, R. D. R., Martinelli Junior, C. E., \& Tourinho Filho, H. (2020). Exergames reduces triceps and subscapular skinfolds and improve self-esteem and body image of obese children. International Journal of Physical Education, Sports and Health, 7 (4), 232-238. https://www.kheljournal.com/archives/2020/vol7issue4/PartD/7-4-24-801.pdf

Barbosa, M. L. M., Barbosa, L. F. M., Vieira, C. J. G., Costa, A. D. P. V., Lima, L. R. A., \& Medeiros, M. L. (2021). Programa de atividade física usando vídeo jogos ativos com adolescentes sedentários. Research, Society and Development, 10 (16), e467101624066. https://doi.org/10.33448/rsd-v10i16.24066

Benzing, V., \& Schmidt, M. (2018). Exergaming for children and adolescentes: strengths, weaknesses, opportunities and threats. Journal of Clinical Medicine, 7 (11), 422. https://doi.org/10.3390/jcm7110422

Bittencourt, M. Z., Reategui, E., Radaelli, R., \& Mossmann, J. B. (2018). O potencial dos exergames para desenvolvimento de atitude positiva na Educação Física Escolar. Revista Novas Tecnologias na Educação, 16 (2), 190-199. https://doi.org/10.22456/1679-1916.89260

Brasil. Ministério da Saúde. Secretaria de Atenção Primária à Saúde. Departamento de Promoção da Saúde. (2021). Guia de Atividade Física para a População Brasileira. Brasília: Ministério da Saúde. http://bvsms.saude.gov.br/bvs/publicacoes/guia_atividade_fisica_populacao_brasileira.pdf 
Deutsch, J. E., Bretterm, A., Smith, C., Welsen, J., John, R., Guarrera-Bowby, P., \& Kafri, M. (2011). Nintendo Wii Sports and Wi Fit game analysis validation and application to stroke rehabilitation. Top Stroke Rehabilitation, 18 (6), 701-719. https://doi.org/10.1310/tsr1806-701

Dias, J. D., Tibes, C. M. S., Fonseca, L. M. M., \& Zem-Mascarenhas, S. H. (2017). Uso de serious games para enfrentamento da obesidade infantil: revisão integrativa da literatura. Texto \& Contexto Enfermagem, 26 (1), e3010015. https://doi.org/10.1590/0104-07072017003010015

Ferreira, A. R., \& Francisco, S. J. (2017). Explorando o potencial dos jogos digitais: uma revisão sobre a utilização dos exergames na educação. Revista IberoAmerica de Estudos em Educação, 12 (Esp. 2), 1177-1193. http://dx.doi.org/10.21723/riaee.v12.n.esp.2.10288

Florêncio Júnior, P. G., Paiano, R., \& Costa, A. S. (2020). Isolamento social: consequências físicas e mentais da inatividade física em crianças e adolescentes. Revista Brasileira de Atividade Física e Saúde, 25 (e0115). https://doi.org/10.12820/rbafs.25e0115

González-González, C. S., Del Rio, N. G., Toledo-Delgado, P. A., \& García-Peñalvo, F. J. (2021). Active game-based solutions for the treatment of childhood obesity. Sensors, 21 (4), 1266, 2021. https://doi.org/10.3390/s21041266

Graf, D. L., Pratt, L. V., Hester, C. N., \& Short, K. R. (2009). Playing active vídeo games increases energy expenditure in children. Pediatrics, 124 (2), $534-$ 540. https://doi.org/10.1542/peds.2008-2851

Kowaluk, A., \& Woźniewski, M. (2019). Interactive video games to promote physical activity among healthy children and youths. Pediatria Polska - Polish Journal of Paediatrics, 94 (3), 198-204. https://doi.org/10.5114/polp.2019.86443

Lamboglia, C. M. G. F., Silva, V. T. B. L., Vasconcelos Filho, J. E., Pinheiro, M. H. N. P., Munguba, M. C. S., Silva Júnior, F. V. I., Paula, F. A. R., \& Silva, C. A. B. (2013). Exergaming as a strategic tool in the fight against childhood obesity: a systematic review. Journal of Obesity, 2013 (438364), 1-8. https://doi.org/10.1155/2013/438364

Lin, J. H. (2015). "Just Dance": The effects os exergame feedback and controller use on physical activity and psychological outcomes. Games Health Journal, 4 (3), 183-189. https://doi.org/10.1089/g4h.2014.0092

Lins, N. C. L., Medeiros, C. C. M., Vianna, R. P. T., Melo, F. C. T., Pimentel, M. M., Queiroz, M. G., Macedo, M. A. M., \& Carvalho, D. F. (2020). Impacto do videogame ativo na autopercepção da imagem corporal e no desenvolvimento de comportamentos de risco para compulsão alimentar em adolescentes com excesso de peso. Research, Society and Development, 9 (10), e1619108317. https://doi.org/10.33448/rsd-v9i10.8317

Marin, J. L. O., Alencar, G. P., Lima, L. E. M., Teixeira, C. V. L. S., \& Silva, A. (2019). O treinamento funcional na infância: o que pensam os pais e as crianças? Revista Brasileira de Fisiologia do Exercício, 18 (1), 17-22. http://dx.doi.org/10.33233/rbfe.v18i1.2759

Medeiros, P., Capistrano, R., Zequinão, M. A., Silva, S. A., Beltrame, T. S., \& Cardoso, F. L. (2017). Exergames como ferramenta de aquisição e desenvolvimento de habilidades e capacidades motoras: uma revisão sistemática. Revista Paulista de Pediatria, 35 (4), 464-471. https://doi.org/10.1590/1984$0462 / ; 2017 ; 35 ; 4 ; 00013$

Papalia, D. E., Olds, S. W., \& Feldman, R. D. (2006). Desenvolvimento humano (8th ed.). Artmed.

Peng, W., Crouse, J. C., \& Lin, J. H. (2012). Using active video games for physical activity promotion: a systematic review of the current state of research. Health Education \& Behavior, 40 (2), 171-192. https://doi.org/10.1177/1090198112444956

Pereira, J. C., Rodrigues, M. E., Campos, H. O., \& Amorim, P. R. S. (2012). Exergames como alternativa para o aumento do dispêndio energético: uma revisão sistemática. Revista Brasileira de Atividade Física e Saúde, 17 (5), 332-340. https://rbafs.org.br/RBAFS/article/view/2171

Peres, E. N., Silva, R. C. B., Castro, A. A. M., Lara, S., Cassol, G., Nogueira, R., Fernandes, R. G., \& Serrão Júnior, N. F. (2021). Efeitos de um programa de exergames sobre o equilíbrio postural em usuários de um Centro de Atenção Psicossocial (CAPS II). Research, Society and Development, 10 (2), e38410212454. https://doi.org/10.33448/rsd-v10i2.12454

Ramírez-Granizo, I. A., Ubago-Jiménez, J. L., González-Valero, G., Puertas-Molero, P., \& Román-Mata, S. S. (2020). The effect of physical activity and the use of active video games: exergames in children and adolescents: a systematic review. International Journal of Environmental Research and Public Heatlh, 17 (12), 4243. https://doi.org/10.3390/ijerph17124243

Shayne, R. K.; Fogel, V. A., Miltenberger, R. G., \& Koehler, S. (2012). The effects of exergaming on physical activity in a third-grade physical education class. Journal of Applied Behavior Analysis, 45 (1), 211-215. https://dx.doi.org/10.1901/jaba.2012.45-211

Silva, K. A., Lima, L. E. M., \& Alencar, G. P. (2018). Influência das aulas de movimento e expressão corporal na motricidade global e equilíbrio em crianças de 3 a 5 anos do SESC Escola Horto. Revista Brasileira de Fisiologia do Exercício, 17 (4), 196-204. http://dx.doi.org/10.33233/rbfe.v17i4.1919

Silva, P. F., Fagundes, L. C., \& Menezes, C. S. (2018). Como as crianças estão se apropriando das Tecnologias Digitais na Primeira Infância? Revista Novas Tecnologias na Educação, 16 (1), 1-10. https://doi.org/10.22456/1679-1916.86023

Vaghetti, C. A. O., Ferreira, E. T., Cavalli, A. S., Monteiro-Junior, R. S., \& Del Vecchio, F. B. (2017). Exergames e sua utilização no currículo escolar: uma revisão sistemática. ConScientiae Saúde, 16 (2), 293-301. https://periodicos.uninove.br/saude/article/view/7189

Valeriani, F., Protano, C., Marotta, D., Liguori, G., Spica, V. R., Valerio, G., Vitali, M., \& Gallè, F. (2021). Exergames in childhood obesity treatment: systematic review. International Journal of Environmental Research and Public Health, 18 (9), 4938. https://dx.doi.org/10.3390\%2Fijerph18094938

Whittemore, R., \& Knafl, K. (2005). The integrative review: updated methodology. Journal of Advanced Nursing, 52 (5), 546-553. https://doi.org/10.1111/j.1365-2648.2005.03621.x

World Health Organization. (2020). Fact Sheet - Physical activity: 26 November 2020. Geneva: WHO. https://www.who.int/news-room/factsheets/detail/physical-activity 